\title{
Genetic Diversity Analysis, Characterization and Evaluation of Elite Chickpea (Cicer arietinum L.) Genotypes
}

\author{
D. K. Janghel*, Krishan Kumar, R. Sunil and A. K. Chhabra \\ Department of Genetics and Plant Breeding, CCS Haryana Agricultural University, \\ Hisar, Haryana-125 004, India \\ *Corresponding author
}

\section{A B S T R A C T}

\section{Keywords}

Chickpea,DUS, PPV\&FRA, profile and Genetic diversity

\section{Article Info}

Accepted:

15 December 2019 Available Online: 20 January 2020
The 60 elite chickpea genotypes were morphologically characterized and classified as per the guidelines for the conduct of DUS test of chickpea (Cicerarietinum L.)by the PPV\&FRA, 2007, GOI. Among 13 DUS traits observed; seven traits were found tri-morphic, four traits di-morphic and two traitspolymorphic among 60 chickpea genotypes indicated the existence of remarkable amount of genetic variability that have great potential to assign distinctive morphological profiles which could be used for varietal identification and characterization. The Non-hierarchical Euclidean cluster analysis using 11 quantitative traits revealed the maximum inter-cluster distances between cluster III \& VII (7.84) and cluster III \& IV (6.84). The genotypes viz., JG-35, JG-315, RSG-931, Vishal, CSJ-741, Vikas, GNG-1958,PG-5, PDG-4, ICCV-6, ICCV-10, JG11 andICCV-2 were found most diverse among 60 chickpea genotypes which could be used as parents in hybridization programme for more heterotic response and generation of better segregants in chickpea breeding.

\section{Introduction}

Chickpea (Cicer arietinum L.), commonly known as gram or Bengal gram, is the third most important pulse crop after common beanPhaseolus vulgaris L. and field pea- Pisum sativum L. (Aggarwal et al., 2015).Chickpea seed is a good source of carbohydrates and proteins, which collectively constitute $80 \%$ of the total dry seed weight (Aggarwal et al., 2013). It also contains on an average of $22 \%$ protein, $63 \%$ carbohydrates, $4.5 \%$ fat, $8 \%$ crude fiber and $2.7 \%$ ash (Wood and Grusak, 2007) as well as minerals(calcium, magnesium, zinc, potassium, iron and phosphorus) and vitamins especially thiamine and niacin (Jukantiet al., 2012). Moreover, its pod shells and seed coats are used as fodder 
(Tahir and Karim, 2011). The assessment of genetic diversity is important not only for crop improvements but also efficient management and conservation of germplasm resources. Varietal descriptions provided by the concerned plant breeders are generally inadequate to characterize a variety.

Therefore, there is a great need to characterize the genotypes and to identify the varietal purity. Identification and characterization of new cultivars are also essential for their efficient utilization and germplasm conservation in any crop improvement programme.

Since, a variety attains acceptance only when farmers get genetically pure seeds of high standards. Characterization of varieties is great significance for the protection of IPR (intellectual property right) as well as quality seed production and certification. It's simple, cheap and do not require any sophisticated laboratory techniques.

However, the generation of data is liable to influenced by a complex G X E interaction, which can be overcome by developing and publishing DUS descriptors, skillful and experienced breeder through awareness about trait diversity. Genetic diversity is the basic requirement of any breeding programme aimed at genetic amelioration of yield (Shafique et al., 2016).It isa pre-requisite in effective hybridization programme for the selection of parents intended to more heterotic responses and potential source of better segregants in subsequent generations for various characters (Dwevedi and Lal, 2009).

Modern plant breeding and agricultural systems have narrowed the base for the genetic diversity of cultivated chickpea. Therefore, it is time to explore new sources of variation that might be used in plant breeding programmes.

\section{Materials and Methods}

The experimental material comprised of 60 elite chickpea genotypes (Table1) from chickpea germplasm maintained at Pulses Section, Department of Genetics and Plant Breeding, CCS Haryana Agricultural University, Hisar (Haryana, India). These genotypes were evaluated in Randomized Block Design (RBD) with three replications during Rabi 2014-15following the recommended agronomical package of practices. The observations were recorded on13 DUS traits for morphological characterization and classification of chickpea genotypes as per the guidelines for the conduct of test for Distinctiveness, Uniformity and Stability (DUS) on chickpea (Cicer arietinum L.) approved by the Protection of Plant Varieties and Farmers' Rights Authority (PPV\&FRA) in 2007, Government of India (Table 2) as well as 11 quantitative traits (days to $50 \%$ flowering, days to maturity, number of secondary branches per plant, number of pods per plant, plant height $(\mathrm{cm})$, number of seeds per pod, 100 seed weight $(\mathrm{g})$, seed yield per plant $(\mathrm{g})$, standard germination $(\%)$, seedling length $(\mathrm{cm})$ and seed vigour index-I) to assess the genetic divergence by Non-hierarchical Euclidean cluster analysis (Spark, 1973) based on Ward's minimum variance method using INDOSTAT software.

\section{Morphological characterization}

Anthocyanin pigmentation on stem is one of most important morphological markers in chickpea germplasm and discriminated into two groups based on their presence or absence. The pigmentation was present in all the Desi types (45 genotypes) and absent in the Kabuli types of chickpea (15 genotypes). Chickpea cultivars were classified into three groups based on plant growth habit viz., erect, semi-erect and spreading (Table 3). The Desi types exhibited all types of growth habit, 
whereas, Kabuli types semi-erect and spreading types of growth habit (Plate- 1). Both the traits were found distinct, uniform and stable in chickpea cultivars that could be used in varietal identifications as well as characterization of chickpea germplasm for their conservation.

The chickpea genotypes were classified into four groups based on foliage colour, viz., Light green, Medium green, Dark green and Greenish purple (Table 4).Only first three types of foliage colour were observed in 60 chickpea genotypes. Based on leaflet size, the chickpea genotypes were classified into three categories viz., small $(<10 \mathrm{~mm})$, medium (10$15 \mathrm{~mm})$ and large $(>15 \mathrm{~mm})$. The Desi types constituted small to large types, while, medium to large in the Kabuli types (Table 5). Both the leaf traits(foliage colour and leaflet size) were quite useful in the characterization of chickpea germplasm but more affected by environmental factors due to the polygenic control and more GX E interactions.

On the basis of number of flowers per peduncle, all the chickpea genotypes were classified into two groups. The Kabuli types had single flower per peduncle and in the Desi types only two genotypes (RSG-931 and CSJ741) showed twin number of flowers per peduncle and remaining others had single flower per peduncle (Plate- 2). On the basis of flower colour, the chickpea genotypes were classified into three groups viz., pink, white and blue colour. The flower colour observed as pink in all the 45 Desi genotypes and white in all the 15 Kabuli genotypes (Plate- 3). Flower colour is reliable morphological marker and distinguishing the chickpea genotypes into two main types. The Kabuli types always exhibited white flower colour which could be typically used for varietal identification. The chickpea genotypes were classified into two categories on the basis of absence or presence of stripes on flower. All the Desi types had stripes on flower (45 genotypes), whereas, the Kabuli types were without stripes (15 genotypes). On the basis of peduncle length, the chickpea genotypes were classified into three main categories presented in Table $6 \mathrm{viz}$., short $(<5 \mathrm{~mm})$, medium $(5-$ $10 \mathrm{~mm})$ and long $(>10 \mathrm{~mm})$. Most of the genotypes were having medium peduncle length. The flower characteristics are most important morphological marker to distinguish the two types of chickpea.

On the basis of pod size, the 60 chickpea genotypes were classified into three categories viz., small $(<15 \mathrm{~mm})$, medium $(15-20 \mathrm{~mm})$ and large $(>20 \mathrm{~mm})$. The Desi types had small to large type, while, the Kabuli type showed medium to large in pod size. Most of the genotypes showed medium to large pod size (Table 7). Chickpea pod size was found to variable due to polygenic control and, genotypic and environmental interactions.

Chickpea cultivars were classified into different groups based on seed colour, seed size, seed testa texture and seed type. On the basis of seed colour, all the 60 genotypes were categorized into seven groups presented in Table 8. Based on seed shape, the 60 chickpea genotypes were classified into three groups (Angular, owl's \& pea shapes). The seed shape was observed as angular in 38 genotypes, owl's shape in 22 genotypes and none was found in pea shaped (Plate-4). On the basis of seed testa texture, the chickpea genotypes were classified into three groups viz., rough, smooth and tuberculated. Most of the genotypes showed rough seed testa texture except two Kabuli types (BG-1053 and IPCK 10-151) which exhibited smooth texture and no genotype was found in tuberculated texture (Plate- 5).Among 60 chickpea genotypes studied, 45 genotypes were classified as the Desi types and 15 genotypes as the Kabuli types based on the seed type (Plate- 6).With respect to seed characteristics, results are 
consistent with the results obtained earlier in chickpea.

\section{Genetic Diversity analysis}

The Non-hierarchical Euclidean cluster analysis grouped 60 chickpea genotypes into seven distinct clusters. Clustering pattern revealed that cluster VI was the largest group (13 genotypes) and cluster VII (4 genotypes)was smallest (Fig. 1). The maximum intra-cluster distances were shown by cluster III (3.71) and cluster VI (3.54), while, minimum by cluster VII (2.92) and cluster V (2.39) in Table 9. The maximum inter-cluster distance were displayed between cluster III \& VII (7.84) followed by cluster III \& IV (6.84), while, minimum by cluster II \& V (3.57), followed by clusters IV \& V (3.81) in Table 9.

The cluster means of chickpea genotypes for 11 quantitative traits revealed considerable differences among all the clusters (Table 10). It was evident that cluster VII had the highest mean value for number of seeds per pod (1.53), germination per cent (85.33), seedling length (30.44) and seed vigour index-I (2664.40), whereas, lowest for morphophenological traits viz., days to $50 \%$ flowering (77.08) and days to maturity (126.00). Cluster IV showed the maximum mean value for number of secondary branches per plant (8.11), number of pods per plant (71.52) and seed yield per plant (19.87). The above comparison indicated that traits of cluster IV and VII had the highest mean value for most of the yield contributing traits. Hence, the genotypes included in the cluster IV (GNG1958, PG-5, PDG-4, BG-3028, \& IPCK 201092) and cluster VII (ICCV-6, ICCV-10, JG-11 \& ICCV-2) were more divergent than the other clusters. Among the 13 morphological DUS traits observed; seven were tri-morphic viz., plant growth habit, leaflet size, flower colour, peduncle length, pod size, seed shape and seed testa texture, four traits dimorphicviz., anthocyanin pigmentation on stem, number of flowers per peduncle, stripes on flower and seed type, and two traits polymorphic viz., foliage colour and seed colour among the 60 elite chickpea genotypes indicated the existence of remarkable amount of genetic variability in these genotypes that have great potential to assign distinctive morphological profiles from combination of morphological DUS traits which could be used for varietal identification and characterization as well as selection of diverse parents in hybridization programme for more heterotic response and generation of better segregants in chickpea breeding. The anthocyanin pigmentation on stem, flower characteristics as well as seed characteristics were able to distinguish chickpea genotypes distinctively, found uniformly, consistently and least affected by environmental factors which could be used for assigning a distinctive morphological profile for germplasm characterization in chickpea. Similar results were described by Archaket al., 2016 for 12 qualitative traits in chickpea viz., early plant vigor, plant growth habit, plant pigmentation, number of leaflets per leaf, leaflet size, plant pubescence, flower color, biomass, pod shape, seed color, seed shape and seed surface.

Non-hierarchical Euclidean cluster analysis using Ward's minimum variance method grouped 60 chickpea genotypes into nonoverlapping seven distinct clusters indicated the presence of substantial amount of genetic diversity among these genotypes. The genotypes of higher intra- and inter-cluster distances have more genetic divergence than the genotypes of minimum intra- and intercluster distances. Therefore, the genotypes of cluster III \& VII and cluster III \& IV could be used as parents in chickpea hybridization programme for obtaining high heterotic response and high frequency of desirable recombinants in the segregating generations. 
Table.1 List of 60 elite chickpea genotypes used for morphological DUS characterization and genetic diversity analysis

\begin{tabular}{|c|c|c|c|c|c|}
\hline SN & Genotypes & Developed by & SN & Genotypes & Developed by \\
\hline 1. & BG 372 & IARI, New Delhi & 31. & RSG 963 & RARI, Durgapura \\
\hline 2. & C 235 & PAU, Ludhiana & 32. & WR 315 & Maintained at CCS HAU \\
\hline 3. & CSG 8962 & CSSRI, Karnal & 33. & DKG 876 & CSK HPK, Dhaulakuan \\
\hline 4. & DCP 92-3 & IIPR, Kanpur & 34. & E $100 Y m$ & CCSHAU, Hisar \\
\hline 5. & GNG 663 & ARS, RAU, Sriganganagar & 35. & H 00-256 & CCSHAU, Hisar \\
\hline 6. & GNG 1581 & ARS, RAU, Sriganganagar & 36. & H 04-99 & CCSHAU, Hisar \\
\hline 7. & GNG 1958 & ARS, RAU, Sriganganagar & 37. & H 07-157 & CCSHAU, Hisar \\
\hline 8. & GNG 1999 & ARS, RAU, Sriganganagar & 38. & H 08-18 & CCSHAU, Hisar \\
\hline 9. & Gaurav & CCSHAU, Hisar & 39. & Н 09-96 & CCSHAU, Hisar \\
\hline 10. & $\mathrm{H}-208$ & CCSHAU, Hisar & 40. & H $10-57$ & CCSHAU, Hisar \\
\hline 11. & HC-1 & CCSHAU, Hisar & 41. & Rajas & MPKV, Rahuri \\
\hline 12. & $\mathrm{HC}-3$ & CCSHAU, Hisar & 42. & Vishal & MPKV, Rahuri \\
\hline 13. & HC-5 & CCSHAU, Hisar & 43. & Vikas & MPKV, Rahuri \\
\hline 14. & Hima & Maintained at CCS HAU & 44. & GNG 2146 & ARS, RAU, Sriganganagar \\
\hline 15. & ICC 4958 & ICRISAT, Hyderabad & 45. & CSJ 741 & RARI, Durgapura \\
\hline 16. & ICCV 6 & IIPR, Kanpur & 46. & BG 1053 & IARI, New Delhi \\
\hline 17. & ICCV 10 & ICRISAT, Hyderabad & 47. & BG 3028 & IARI, New Delhi \\
\hline 18. & JG 11 & JNKVV, Jabalpur & 48. & GNG 1969 & ARS, RAU, Sriganganagar \\
\hline 19. & JG 35 & JNKVV, Jabalpur & 49. & HK 1 & CCSHAU, Hisar \\
\hline 20. & JG 315 & JNKVV, Jabalpur & 50. & HK 2 & CCSHAU, Hisar \\
\hline 21. & Katila & Maintained at CCS HAU & 51. & HK 4 & CCSHAU, Hisar \\
\hline 22. & NARC 9006 & Maintained at CCS HAU & 52. & ICCV 2 & ICRISAT, Hyderabad \\
\hline 23. & Pusa 547 & IARI, New Delhi & 53. & JGK 1 & JNKVV, Jabalpur \\
\hline 24. & PBG 5 & PAU, Ludhiana & 54. & JGK 27 & JNKVV, Jabalpur \\
\hline 25. & PDG 4 & PAU, Ludhiana & 55. & L $550(\mathrm{~K})$ & PAU, Ludhiana \\
\hline 26. & PDG 84-16 & PAU, Ludhiana & 56. & Virat & MPKV, Rahuri \\
\hline 27. & PG 5 & MPKV, Rahuri & 57. & GNG 2237 & ARS, RAU, Sriganganagar \\
\hline 28. & PG 517 & MPKV, Rahuri & 58. & HK 07-234 & CCSHAU, Hisar \\
\hline 29. & RSG 888 & RARI, Durgapura & 59. & IPCK 2010-92 & IIPR, Kanpur \\
\hline 30. & RSG 931 & RARI, Durgapura & 60. & IPCK 10-151 & IIPR, Kanpur \\
\hline
\end{tabular}

Table.2 Guidelines for DUS test by PPV\&FRA, 2007 for 13 morphological DUS traits on chickpea

\begin{tabular}{|c|c|c|c|}
\hline SN & Characteristics & States & Observation stage \\
\hline 1. & $\begin{array}{l}\text { Anthocyanin pigmentation } \\
\text { on stem }\end{array}$ & Absent/ Present. & Before flowering \\
\hline 2. & Plant growth habit & $\begin{array}{l}\text { Erect }\left(0-15^{\circ} \text { from vertical }\right) / \text { Semi-erect }\left(16-60^{\circ} \text { from vertical }\right) / \text { Spreading } \\
\text { (61-80 from vertical). }\end{array}$ & \multirow[t]{6}{*}{ At $50 \%$ flowering } \\
\hline 3. & Foliage colour & Light green/ Medium green/ Dark green/ Greenish purple. & \\
\hline 4. & Leaflet size (Length) & Small $(<10 \mathrm{~mm}) /$ Medium $(10-15 \mathrm{~mm}) /$ Large $(>15 \mathrm{~mm})$ & \\
\hline 5. & $\begin{array}{l}\text { Number of flowers/ } \\
\text { peduncle }\end{array}$ & Single/ Twin. & \\
\hline 6. & Flower colour & White/ Pink/ Blue. & \\
\hline 7. & Stripes on flower & Absent/ Present. & \\
\hline 8. & Peduncle length & Short $(<5 \mathrm{~mm}) /$ Medium $(5-10 \mathrm{~mm}) /$ Long $(>10 \mathrm{~mm})$. & Pod development \\
\hline 9. & Pod size & Small $(<15 \mathrm{~mm}) /$ Medium $(15-20 \mathrm{~mm}) /$ Large $(>20 \mathrm{~mm})$. & Harvest maturity \\
\hline 10. & Seed colour & $\begin{array}{c}\text { Beige (Kabuli)/ Creamy beige/ Green/ yellow/ Orange brown/ Dark brown/ } \\
\text { Grey black. }\end{array}$ & \multirow[t]{4}{*}{30 days after harvest } \\
\hline 11. & Seed shape & Pea-shaped/ Owl's head/ Angular. & \\
\hline 12. & Seed testa texture & Rough/ Smooth/ Tuberculated. & \\
\hline 13. & Seed type & Desil Kabuli. & \\
\hline
\end{tabular}


Table.3 Classification of 60 chickpea genotypes on the basis of plant growth habit

\begin{tabular}{|c|c|}
\hline Erect (6) & GNG-1958, Gaurav, HC-5, DKG-876, H 09-96, GNG-2146. \\
\hline Semi-erect & BG-372, C-235, CSG-8962, DCP 92-3, GNG-663, GNG-1581, GNG-1999, H-208, HC-3, ICCV- \\
$\mathbf{( 4 0 )}$ & 10, JG-11, JG-35, JG-315, NARC-9006, Pusa-547, PBG-5, PDG-4, PG-5, RSG-888, RSG- \\
& 931,RSG-963, E-100Ym, H 00-256, H 04-99, H 07-157, H 10-57, Rajas, Vishal, Vikas, CSJ- \\
& 741, BG-1053, BG-3028, GNG-1969, HK-1, HK-2, HK-4, ICCV-2, JGK-27, Virat, GNG-2237. \\
\hline $\begin{array}{c}\text { Spreading } \\
(\mathbf{1 4})\end{array}$ & HC-1, Hima, ICC-4958, ICCV-6, Katila, PDG 84-16, PG-517, WR-315, H 08-18, JGK-1, L-550, \\
\hline
\end{tabular}

Table.4 Classifications of 60 chickpea genotypes on the basis of foliage colour

\begin{tabular}{|c|c|}
\hline Light green (6) & BG-372, GNG-663, Gaurav, PDG 84-16, CSJ-741, BG-3028. \\
\hline $\begin{array}{c}\text { Medium green } \\
\text { (19) }\end{array}$ & $\begin{array}{c}\text { DCP 92-3, HC-3, Hima, ICC-4958, JG-315, RSG-963, WR-315, E-100Ym, H 00-256, H 04- } \\
\text { 99, H 10-57, Rajas, Vishal, GNG-1969, HK-2, ICCV-2, JGK-27, L-550, IPCK-2010-92. }\end{array}$ \\
\hline $\begin{array}{c}\text { Dark green } \\
\text { (35) }\end{array}$ & $\begin{array}{c}\text { C-235, CSG-8962, GNG-1581, GNG-1958, GNG-1999, H-208, HC-1, HC-5, ICCV-6, ICCV- } \\
\text { 10, JG-11, JG-35, Katila, NARC-9006, Pusa-547, PBG-5, PDG-4, PG-5, PG-517, RSG-888, } \\
\text { RSG-931, DKG-876, H 07-157, H 08-18, H 09-96, Vikas, GNG-2146, BG-1053, HK-1, HK- } \\
\text { 4, JGK-1, Virat, GNG-2257, HK 07-234, IPCK 10-151. }\end{array}$ \\
\hline
\end{tabular}

Table.5 Classifications of 60 chickpea genotypes on the basis of leaflet size

\begin{tabular}{|c|c|}
\hline $\begin{array}{l}\text { Small } \\
(22)\end{array}$ & $\begin{array}{l}\text { DCP 92-3, GNG-663, H-208, HC-1, BG-372, C-235, CSG-8962, GNG-1581, GNG-1999, Gaurav, } \\
\text { HC-5, Hima, ICCV-10, JG-315, PDG-4, PDG 84-16, RSG-888, WR-315, H 07-157, H 09-96, H } \\
\text { 10-57, Vikas. }\end{array}$ \\
\hline $\begin{array}{c}\text { Medium } \\
\text { (33) }\end{array}$ & $\begin{array}{l}\text { ICCV-6, PG-517, RSG-931, DKG-876, E-100Ym, Rajas, HC-3, Katila, Pusa-547, PG-5, H 00-256, H } \\
\text { 08-18, GNG-2146, CSJ-741, BG-1053, GNG-1969, HK-4, ICC-4958, JG-11, NARC-9006, PBG-5, } \\
\text { RSG-963, H 04-99, Vishal, BG-3028, HK-1, Virat, GNG-1958, HK-2, ICCV-2, JGK-1, JGK-27, } \\
\text { IPCK 10-151. }\end{array}$ \\
\hline $\begin{array}{l}\text { Large } \\
\text { (5) }\end{array}$ & JG-35, L-550, HK 07-234, IPCK 2010-92, GNG-2237. \\
\hline
\end{tabular}

Table.6 Classification of 60 chickpea genotypes on the basis of peduncle length

\begin{tabular}{|c|c|}
\hline Short (2) & PDG-4, PG-5. \\
\hline Medium (38) & $\begin{array}{l}\text { BG-372, C-235, CSG-8962, H-208, GNG-1958, GNG-1999, Gaurav, H 09-96, DCP 92-3, GNG-663, } \\
\text { GNG-1581, HC-1, HC-5, Hima, ICCV-6, ICCV-10, JG-315, NARC-9006, Pusa-547, RSG-888, RSG-931, } \\
\text { RSG-963, DKG-876, E-100Ym, H 04-99, H 07-157, H 10-57, Vikas, IPCK 10-151, HC-3, PBG-5, PDG } \\
\text { 84-16, PG-517, H 00-256, Vishal, GNG-2146, CSJ-741, BG-1053. }\end{array}$ \\
\hline Long (20) & $\begin{array}{c}\text { ICC-4958, JG-35, WR-315, H 08-18, Rajas, L-550, Virat, JGK-1, JGK-27, JG-11, BG-3028, GNG-1969, } \\
\text { HK-1, HK-4, ICCV-2, HK-2, GNG-2237, Katila, HK 07-234, IPCK 2010-92. }\end{array}$ \\
\hline
\end{tabular}

Table.7 Classification of 60 chickpea genotypes on the basis of pod size

\begin{tabular}{|c|c|}
\hline Small (6) & Hima, BG-372, GNG-1999, H-208, WR-315, GNG-663. \\
\hline $\begin{array}{c}\text { Medium } \\
(\mathbf{3 0})\end{array}$ & $\begin{array}{c}\text { RSG-963, H 10-57, HC-5, H 00-256, C-235, GNG-1958, PDG 84-16, HC-1, CSJ-741, H 09-96, } \\
\text { RSG-888, DCP 92-3, Katila, CSG-8962, RSG-931, H 07-157, PDG-4, GNG-1581, H 08-18, } \\
\text { ICCV-6, Vikas, JG-315, E-100Ym, Gaurav, HC-3, DKG-876, ICC-4958, PG-517, JG-11, Vishal. }\end{array}$ \\
\hline $\begin{array}{c}\text { Large } \\
(\mathbf{2 4})\end{array}$ & $\begin{array}{c}\text { Rajas, H 04-99, GNG-2146, NARC-9006, Pusa-547, PBG-5, ICCV-10, HK-1, IPCK 10-151, } \\
\text { IPCK 2010-92, PG-5, GNG-1969, L-550, Virat, JG-35, ICCV-2, HK 07-234, JGK-27, HK-4, } \\
\text { BG-1053, JGK-1, GNG-2237, HK-2, BG-3028. }\end{array}$ \\
\hline
\end{tabular}


Table.8 Classification of chickpea genotypes on the basis of seed colour

\begin{tabular}{|c|c|}
\hline Creamy (4) & GNG-1969, JGK-1, Virat, IPCK 10-151. \\
\hline Creamy white (11) & $\begin{array}{c}\text { BG-1053, BG-3028, HK-1, HK-2, HK-4, ICCV-2, JGK-27, L-550, GNG-2237, HK } \\
\text { 07-234, IPCK 2010-92. }\end{array}$ \\
\hline $\begin{array}{c}\text { Green (1) } \\
\text { Light reddish brown (13) }\end{array}$ & $\begin{array}{r}\text { C-235, CSG-8962, Gaurav, DCP 92-3, GNG-663, GNG-1581, GNG-1958, JG-315, } \\
\text { Katila, NARC-9006, PDG-4, RSG-888, GNG-2146. }\end{array}$ \\
\hline Reddish brown (12) & $\begin{array}{c}\text { BG-372, ICC-4958, ICCV-10, JG-35, PDG 84-16, PG-5, PG-517, E-100Ym, H 04- } \\
\text { 99, Rajas, Vishal, CSJ-741. }\end{array}$ \\
\hline Light yellowish brown (8) & H 10-57, GNG-1999, RSG-963, WR-315, DKG-876, H 09-96, Vikas, PBG-5. \\
\hline Yellowish brown (11) & H-208, HC-1, HC-3, HC-5, ICCV-6, JG-11, Pusa-547, RSG-931, H 00-256, H 07- \\
\hline \begin{tabular}{c} 
157, H 08-18. \\
\hline
\end{tabular} & \\
\hline
\end{tabular}

Table.9 Intra (diagonal) and Inter-cluster (above diagonal) distances for 60 chickpea genotypes

\begin{tabular}{|c|c|c|c|c|c|c|c|}
\hline & Cluster I & Cluster II & Cluster III & Cluster IV & Cluster V & Cluster VI & Cluster VII \\
\hline Cluster I & $\mathbf{3 . 0 9}$ & 4.27 & 5.59 & 4.84 & 3.72 & 5.58 & 5.71 \\
\hline Cluster II & & $\mathbf{3 . 2 6}$ & 4.35 & 5.74 & 3.57 & 5.00 & 5.83 \\
\hline Cluster III & & & $\mathbf{3 . 7 1}$ & 6.84 & 4.78 & $\mathbf{6 . 1 4}$ & $\mathbf{7 . 8 4}$ \\
\hline Cluster IV & & & & $\mathbf{3 . 0 3}$ & 3.81 & 4.86 & 5.72 \\
\hline Cluster V & & & & & $\mathbf{2 . 3 9}$ & 4.10 & 5.04 \\
\hline Cluster VI & & & & & & $\mathbf{3 . 5 4}$ & 4.72 \\
\hline Cluster VII & & & & & & & $\mathbf{2 . 9 2}$ \\
\hline
\end{tabular}

Table.10 Cluster means of various quantitative traits of chickpea genotypes

\begin{tabular}{|c|c|c|c|c|c|c|c|c|c|c|c|}
\hline Clusters & DF & DM & SB/P & Pd/P & PH & Ss/Pd & $\mathbf{1 0 0 S}$ & SY/P & SG & SL & SVI-I \\
\hline I & 89.94 & 145.49 & 7.64 & 45.52 & 47.88 & 1.51 & 15.11 & 13.72 & 83.52 & 28.95 & 2419.5 \\
\hline II & 88.70 & 142.76 & 6.04 & 37.61 & 56.13 & 1.38 & 17.79 & 10.57 & 79.37 & 29.31 & 2327.0 \\
\hline III & 90.60 & 150.47 & 5.80 & 39.40 & 55.80 & 1.23 & 23.21 & 10.10 & 79.00 & 26.71 & 2110.8 \\
\hline IV & 90.48 & 148.00 & $\mathbf{8 . 1 1}$ & $\mathbf{7 1 . 5 2}$ & 57.82 & 1.43 & 23.27 & $\mathbf{1 9 . 8 7}$ & 85.07 & 30.44 & 2589.8 \\
\hline V & 89.21 & 142.50 & 6.96 & 54.33 & 58.75 & 1.40 & 19.73 & 15.43 & 82.00 & 29.23 & 2396.1 \\
\hline VI & 86.07 & 134.80 & 6.47 & 53.00 & $\mathbf{6 0 . 3 3}$ & 1.37 & $\mathbf{3 3 . 1 7}$ & 12.95 & 83.13 & 30.62 & 2546.1 \\
\hline VII & $\mathbf{7 7 . 0 8}$ & $\mathbf{1 2 6 . 0 0}$ & 5.58 & 53.00 & 56.58 & $\mathbf{1 . 5 3}$ & 20.68 & 14.52 & $\mathbf{8 5 . 3 3}$ & $\mathbf{3 1 . 2 1}$ & $\mathbf{2 6 6 4 . 4}$ \\
\hline Mean & 88.43 & 142.87 & 6.75 & 48.83 & 55.57 & 1.41 & 20.31 & 13.61 & 82.02 & 29.42 & 2415.3 \\
\hline
\end{tabular}

DF-Days to 50 percent flowering, DM- Days to maturity, SB/P- Number of secondary branches per plant, Pd/PNumber of pods per plant, PH - Plant height (cm), Ss/Pd- Number of seeds per pod, 100SW (g)-100 seed weight (g), SY/P- Seed yield per plant (g), SG (\%)- Standard germination (\%), SL (cm)- Seedling length (cm), SVI-I- Seed vigour Index-I. 
Plate.1 Plant growth habit

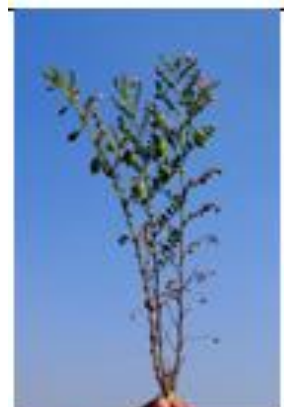

Erect

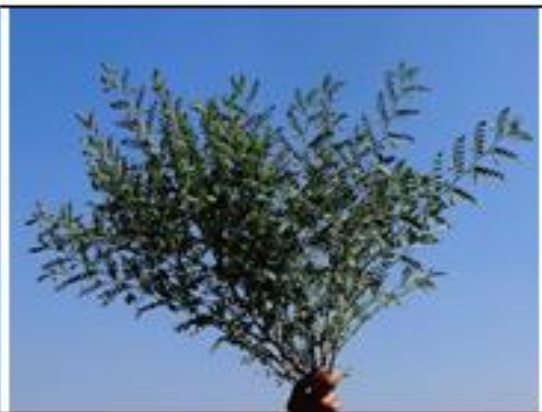

Semi-erect

Plate.2 Number of flowers per peduncle

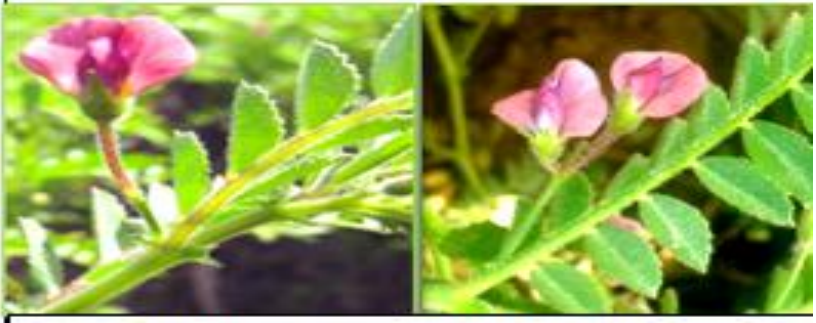

Single flower

Plate.4 Seed shape

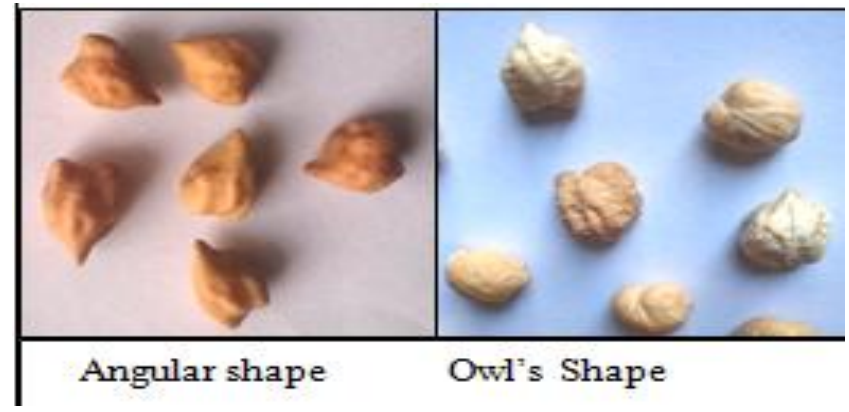

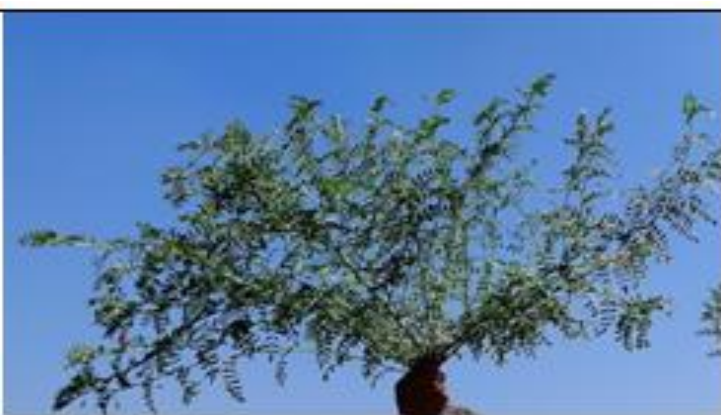

Spreading

Plate.3 Flower colour

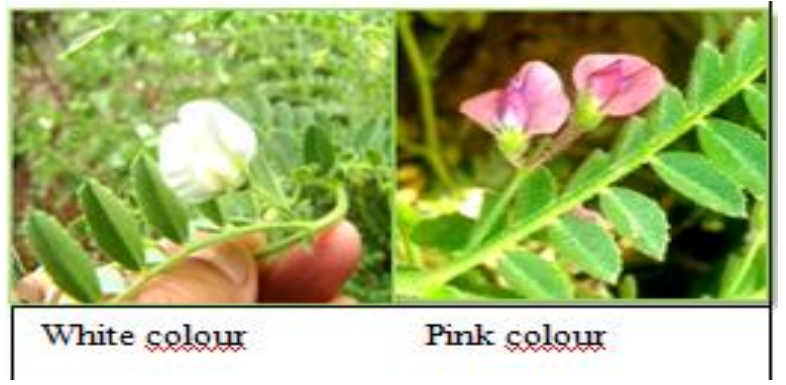

Plate.5 Seed texta textures

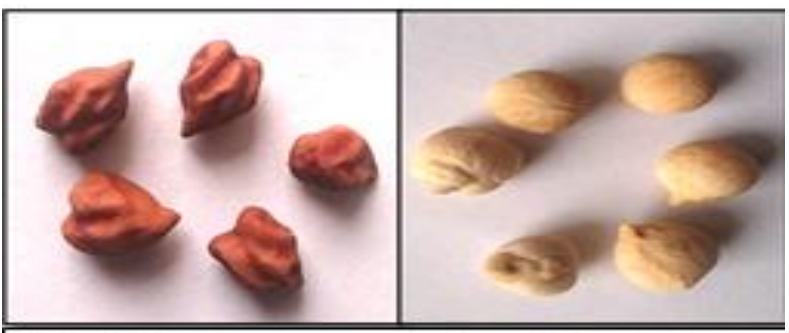

Rough texture

Smooth texture

Plate.6 Seed types

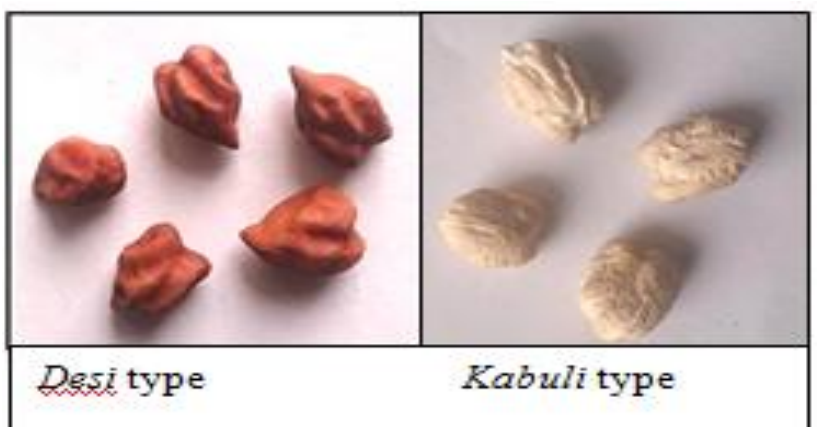


Fig.1 Dendrogram showing clustering pattern of 60 elite chickpea genotypes for seed yield and its component trait
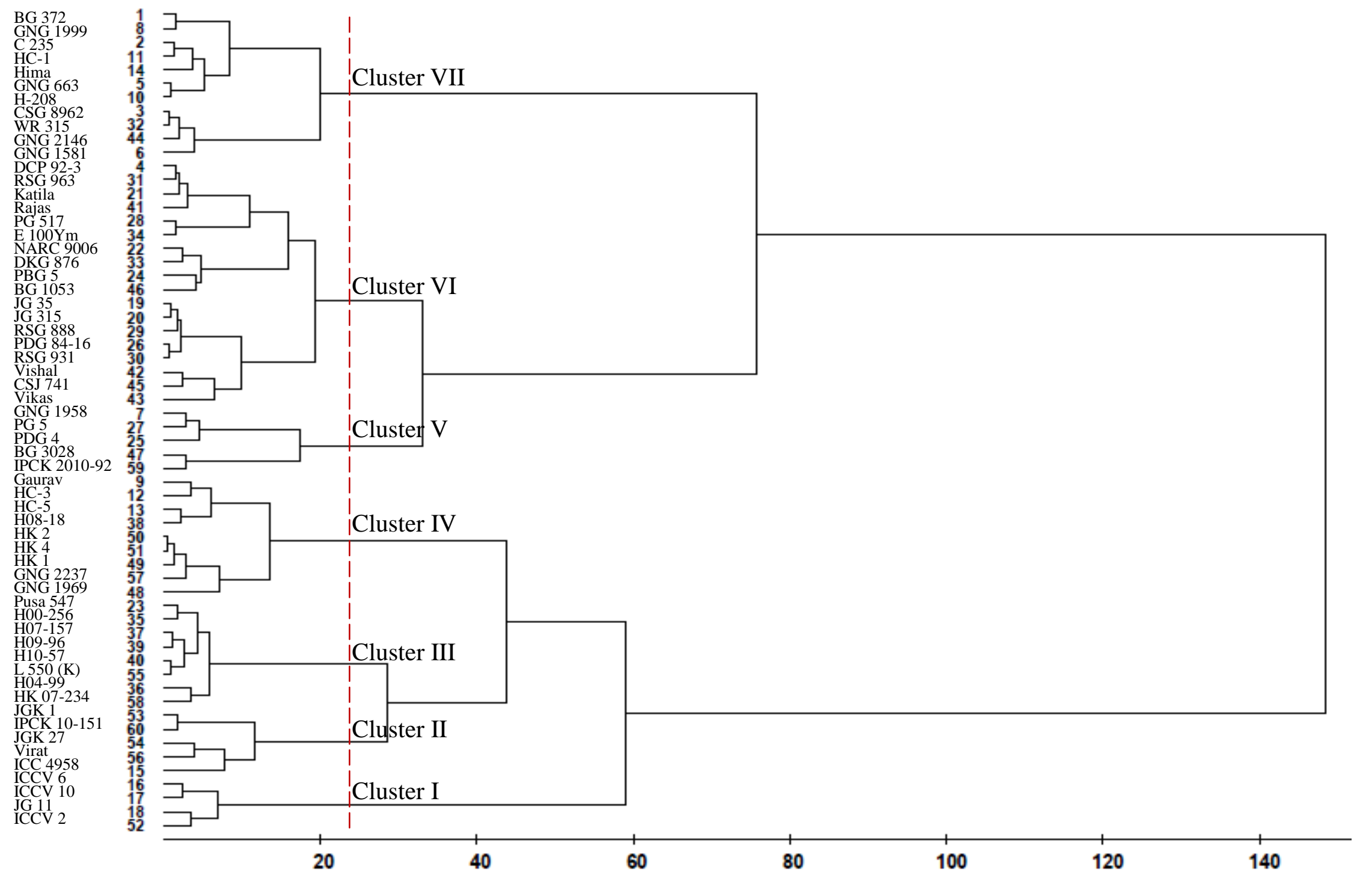
The genotypes of cluster III, IV and VII (JG35, JG-315, RSG-888, PDG 84-16, RSG-931, Vishal, CSJ-741, Vikas; GNG-1958,PG-5, PDG-4, BG-3028, IPCK 2010-92; ICCV-6, ICCV-10, JG-11 andICCV-2, respectively) had highest inter-cluster distances as well as higher cluster means for most of the yield component traits indicated that these genotypes were most diverse among 60 elite chickpea genotypes which could be used as parents in hybridization programme of chickpea breeding. The above findings are broadly in agreement with the report of Dwevedi and Lal, 2009; Yadavet al., 2010 and Ojhaet al., 2011.

Genetic diversity analysis and morphological DUS characterization among 60 chickpea genotypes were differed significantly for 13 morphological DUS traits and 11 quantitative traits, indicating a large and exploitable amount of genetic variability in these genotypes. The morphological DUS descriptors viz., anthocyanin pigmentation on stem, flower characteristics as well as seed characteristics were able to distinguish chickpea genotypes distinctively, uniformly and consistently, and least affected by environmental factors which could be used for assigning a distinctive morphological profile for germplasm characterization in chickpea. Thus, characterization of varieties is in great significance for the identity of a variety, germplasm conservation, protection of IPR rights, maintenance of genetic purity of seed lots and comparisons of breeding lines (Inbreds, purelines and hybrids) for the practical application of plant breeding in the field. Genetic diversity analysis based on Nonhierarchical Euclidean cluster analysis also displayed substantial amount of genetic diversity in the chickpea genotypes viz., JG35, JG-315, RSG-888, PDG 84-16, RSG-931, Vishal, CSJ-741, Vikas, GNG-1958, PG-5, PDG-4, BG-3028, IPCK 2010-92; ICCV-6, ICCV-10, JG-11 and ICCV-2 which could be used in future breeding programme of chickpea for development of elite breeding materials.

\section{Acknowledgement}

The authors are thankful of Dr. S.S. Verma, Principal Scientist, Department of Seed Science and Technology for their guidance and support throughout the research period.

\section{References}

Aggarwal, H., Rao, A., Rana, J.S., Singh, J., Kumar, A., Chhokar, V. and Beniwal, V. (2015). Assessment of genetic diversity among 125 cultivars of chickpea (Cicer arietinum L.) of Indian origin using ISSR markers.Turkish Journal of Botany, 39: 218-226.

Aggarwal, Nagargoje, S., Dhakne, K., Chavhan, R.L., Hinge, V.R. and Dethe, A.M. (2016). Genetic diversity study among chickpea genotypes exploiting RAPD and SSR markers. Asian Journal of Multidisciplinary Studies, 2(6): 141-155.

Archak, S., Tyagi, R.K., Harer, P.N., Mahase, L.B., Singh, N., Dahiya, O.P., Nizar M.A., Singh, M., Tilekar, V., Kumar, V., Dutta, M., Singh, N.P. and Bansal, K.C. (2016). Characterization of chickpea germplasm conserved in the Indian National Genebank and development of a core set using qualitative and quantitative trait data. The Crop Journal, 189: 1-8.

Dwevedi, K.K. and Lal, G.M. (2009). Assessment of genetic diversity of cultivated chickpea (Cicerarietinum L.). Asian Journal of Agricultural Sciences, 1: 7-8.

Jukanti, A.K., Gaur, P.M., Gowda, C.L.L. and Chibbar, R.N. (2012).Nutritional quality and health benefits of chickpea 
(Cicer arietinum L.).British Journal of Nutrition, 108: S11-S26.

Ojha, V.S., Shiva, N. and Singh, R. (2011).Genetic variability in chickpea (Cicer arietinum L.).Progressive Research, 5(2): 275-276.

Shafique, M.S., Ahsan, M., Mehmood, Z., Abdullah, M., Shakoor, A. and Ahmad, M.I. (2016).Genetic variability and inter-relationship of various agronomic traits using correlation and path analysis in chickpea (Cicer arietinum L.). Academia Journal of Agricultural Research, 4(2): 82-85.

Spark, D.N. (1973). Euclidean cluster analysis. Algorithm As. 58.Applied Statistics, 22: 126-130.
Tahir, N.A.R. and Karim, H.F.H. (2011).Determination of genetic relationships among some varieties of chickpea (Cicer arietinum L.) in Sulaimani by RAPD and ISSR markers. Jordan Journal of Biological Science, 4: 77-86.

Wood, J.A. and Grusak, M.A. (2007).Nutritional value of chickpea in Chickpea breeding and management. CAB. International, UK, 101-142.

Yadav, A.K., Mishra, S.B., Singh, S.S. and Madhuri, A. (2010). Character association and genetic divergence study in chickpea (Cicerarietinum L.). Environment and Ecology, 28(2B): 1276-1280.

\section{How to cite this article:}

Janghel, D. K., Krishan Kumar, R. Sunil and Chhabra, A. K. 2020. Genetic Diversity Analysis, Characterization and Evaluation of Elite Chickpea (Cicer arietinum L.) Genotypes. Int.J.Curr.Microbiol.App.Sci. 9(01): 199-209. doi: https://doi.org/10.20546/ijcmas.2020.901.023 